\title{
EOPS: Efecto protector de la lactancia sobre la ocurrencia de otitis media aguda en niños
}

Breastfeeding and its protective effect in otitis media occurrence in children

Silvio Torres*

\begin{abstract}
Resumen
Un caso de una madre de una beba de 4 meses que consulta por el posible efecto de la suspensión de la lactancia y la incidencia de otitis es planteado. Se confecciona la pregunta clínica, se describe la estrategia de búsqueda, así como la principal fuente recabada. Dicho estudio prospectivo, que evalúa el desarrollo de otitis media aguda y otitis media con efusión, concluye que la alimentación por fórmula parecería aumentar el riesgo de otitis de los 3 a 12 meses de vida. Si bien la evidencia no es concluyente, tanto este como otros estudios sugieren un beneficio de la lactancia materna en la incidencia de OMA.
\end{abstract}

\section{Abstract}

A mother of a 4 month baby asks about the possible effects of stopping breastfeeding and the incidence of otitis. A clinical question is posed, the search strategy is described, and the most relevant paper is summarized. In that prospective study, which evaluated the development of acute otitis media and acute otitis media with effusion, authors conclude that formula feeding appears to increase otitis risk between 3 to 12 months of age. Though the evidence is not conclusive, this and other studies, suggest that breastfeeding is beneficial regarding otitis incidence.

Palabras clave: Lactancia materna, otitis media aguda. Key words: Breast feeding, Acute otitis media.

Torres S. Evidencia Orientada al Paciente. Lactancia materna y su efecto protector en la ocurrencia de otitis media aguda en niños. Evid. actual. práct. ambul; 11(2): 62-63, Mar-Abr.2008.

\section{Escenario clínico}

Me realizan una consulta pediátrica, una madre, primigesta, con su beba de 4 meses, que concurre al control pediátrico mensual. Se encuentra angustiada, por la merma de su producción de leche, a pesar de todas las técnicas de puericultura empleadas me plantea una posible suspensión de la lactancia en el futuro cercano. Además, plantea la inquietud, obtenida desde los medios de comunicación, acerca de la frecuencia de infecciones en los oídos, en los bebes no alimentados a pecho.

\section{Pregunta que generó el caso}

¿Los niños (población), que no son alimentados a pecho (en relación a los que sí lo son) [exposición/comparación], ¿son más propensos a padecer otitis media aguda (resultado)?

\section{Estrategia de búsqueda}

Se realizó una búsqueda en Medline, utilizando los términos "Breast feeding" y "acute otitis media" combinándolos a través del operador AND.

Inicialmente se intentó restringir la busqueda a revisiones sistemáticas y meta-análisis publicados en los últimos cinco años, pero al no encontrar evidencia se prolongó a 10 y 15 años. Sólo se encontró un meta-análisis, que evalúa los factores de riesgo para otitis media aguda (OMA) en general, sin focalizar en la lactancia materna. (Clinical Infect Disease,1996;22:10791082). Al ampliar la búsqueda a otro tipo de diseño, la mejor evidencia que he recabado, es un importante estudio de cohorte $^{\star}$, de 1997, que he considerado analizar.

\section{Lactancia y OMA}

La aseveración acerca de los efectos protectores de la lactancia materna en el desarrollo de otitis media aguda, forma parte de todas las publicaciones y cursos que habitualmente se imparten en los consultorios de perinatología y puericultura. Siempre se ha asumido como una verdad revelada, dictaminada por los especialistas experimentados en pediatría ambulatoria, hasta el punto de ser base en la recomendación de la SAP
(Sociedad Argentina de Pediatría). Los mecanismos, que se exponen, para la protección se basan en mecanismos inmunológicos (vía Inmunoglobulina A) y no inmunológicos, tales como la exposición temprana oral del bebe a microorganismos, mejoramiento de la nutrición, transferencia de elementos bioactivos y antibacterianos. Sin embargo, siempre se concluye que deben realizarse estudios confirmatorios, a todos estos supuestos.

\section{Resumen de la Evidencia}

Titulo: "Exclusive Breastfeeding Protects Against Bacterial Colonization and Day Care Exposure to Otitis Media". Pediatrics, Vol 100, ${ }^{\circ} 4$, October 1997. Autores: L Duffy, H Faden, R Wasielewski, y col.

Objetivo: Demostrar la relación entre lactancia materna y otitis media aguda / otitis media con efusión o derrame, en el seguimiento de una cohorte de 306 pacientes, en dos centros pediátricos.

\section{Diseño: Estudio de cohorte prospectivo*.}

Material y Métodos: Se trata de una cohorte de 306 pacientes, se realizó una base de datos con todos los pacientes que consultaron a control pediátrico, a dos centros pediátricos, dependientes de la Universidad de Buffalo (N.Y, EE.UU.).

Se dividieron dos grupos, lactancia materna (exposición) y lactancia con formula (no exposición), se consignaron los datos por mes, los primeros 6 meses, y luego a los 8,10,12,18 y 24 meses. El análisis de datos desde los 12 meses, fue comparando los grupos formula exclusiva contra pecho y alimentación (Grupo mixto ) debido a la incorporación de la alimentación sólida - semisólida, desde los 6 meses.

En los registros, se consignaron edad materna, nivel educacional y sociocultural, existencia de fumadores en el hogar, y colonización de nasofaringe bacteriana (confirmado por cultivo). Los diagnósticos de Otitis media Aguda (OMA) y Otitis Media con Efusión (OME), se realizaron por otoscopia neumática y timpanometría.

Medición de Resultados: El resultado primario a evaluar fue la frecuencia de OMA / OME, a los 3-6-12 y 24 meses. Se cuantificó el Riesgo Relativo e Intervalo de Confianza en los grupos de la cohorte.

* Médico Pediatra, Hospital Universitario Austral. silviot@ fibertel.com.ar 
A partir de los datos reportados por los autores he calculado las reducciones de riesgo absolutas, y el número necesario a tratar (que expresaría el número de bebes que deben tomar pecho para evitar una OMA).

Resultados: En la cohorte de 306 pacientes, culminaron el seguimiento a los 2 años 258 pacientes (Pérdida de seguimiento: $15 \%)$.

Tabla: Resultados principales del análisis univariado*.

\begin{tabular}{c|c}
\hline Seguimiento & RR Fórmula / Pecho (IC95) \\
\hline Tres meses & $1,22(0,97$ a 1,54$)$ \\
\hline Seis meses & $1,59(1,13$ a 2,24$)$ \\
\hline $\mathbf{1 2}$ meses@ & $1,30(0,99$ a 1,70$)$ \\
\hline
\end{tabular}

^Aquí la comparación es fórmula vs. Pecho más alimentación.

Los episodios de OMA/OME, durante el segundo año de vida, que incluyen los episodios recurrentes, mostraron los siguientes valores:

Formula vs. Pecho+Alimentación: RR 1,35 (IC95 0,82 a 2,22) para OMA, y RR 1,65(IC95 0,67 a 4,07) para OME.

Cuando se ajustó* por la presencia de colonización positiva en nasofaringe, las medidas a los tres meses mostraron una mayor asociación entre ausencia de lactancia materna y desarrollo de OM (RR 1,62, IC95 1,12 a 2,30) y a los 6 meses (RR 2,17; IC95 1,36 a 3,48).

En un análisis multivariado* incluyendo potenciales confundidores, la alimentación por fórmula fue el mayor predictor de desarrollo de OMA u OME, y la lactancia materna, incluso por períodos cortos (tres meses) confirió un efecto protector.

Adaptando estos resultados para evaluar su potencial impacto absoluto, la reducción de riesgo absoluto por la alimentación materna fue de alredor del $25 \%$ a los tres y seis meses, lo que implicaría una Número Necesario a Tratar de 4 niños.

\section{Conclusión}

Los autores reportan, que al menos en los primeros 3-6 meses, la lactancia materna tendría un efecto protector, sobre la ocurrencia de OMA y OME. Se informa que el pico de incidencia de OMA en el grupo de los alimentados a fórmula, se manifiesta entre los 3 a 6 meses, y en los alimentados a pecho exclusivo, al año de edad.

\section{Comentario del artículo}

Los estudios acerca de los beneficios de la leche materna exclusiva sobre la OMA no tienen una sólida evidencia, si embargo analizamos una cohorte de 306 pacientes, con una perdida de seguimiento pequeña, menor a $20 \%$, y cuya distribución de grupos fue homogénea. No parecería tampoco haber sesgos de información ya que el método diagnóstico utilizado, aunque subjetivo, es el método estándar.

A pesar de que los resultados univariados* no son significativos, estos si arrojan una asociación significativa y relevante entre lactancia y otitis al ajustarse por potenciales confundidores, concluyendo que la alimentación por fórmula duplica el riesgo de OMA u OMAE a los seis meses de edad.

Los pacientes del estudio son similares al paciente presentado en el caso, en cuanto a etnia, inserción sociocultural y económica de la madre, edad materna, y falta de antecedentes patológicos en el bebé.

También los autores refieren que sus resultados son coincidentes con resultados de metanálisis recientes realizados.

Por lo tanto, creo que si bien habría un efecto beneficioso, también se puede aclarar a la madre que las otitis en los niños son muy frecuentes, y en su mayoría sin un impacto relevante o secuelas. De este modo podemos aconsejar mejor e informarla para ayudar a la toma de decisiones.

\section{Comentario Final}

Si bien la evidencia no es concluyente, tanto este como otros estudios sugieren un beneficio de la lactancia materna en la incidencia de OMA. De todos modos, no debemos preocupar a la madre, y comentarle que las otitis son algo muy frecuente y en general autolimitadas. También debemos hablarle de que hay otros factores más determinantes, tal como la exposición al tabaco en el hogar, la concurrencia a guarderías, la falta de inmunizaciones contra haemophilus y neumococo, entre otros. El objetivo, es poder contener a la madre primeriza, mermando su angustia, y pudiendo contar que parece haber una diferencia en cuanto a la propensión de otitis y lactancia, pero que eso sólo es uno de los elementos de la decisión.

Ver glosario* 